\title{
A New Emotional Classification Method Based on the Combined Characteristics of EEG Signals
}

\author{
Tengfei Wang a , Guangyuan Liu* \\ Southwest University, Chongqing 400700, China; \\ 5527196208@qq.com, ${ }^{*}$ liugy@swu.edu.cn
}

Keywords: EEG Signals, A New Emotional, emotional classification

Abstract: The electroencephalogram (EEG) signals has been widely used in emotion recognition. The entropy features are often used in the emotional recognition using EEG. However, its recognition accuracy remains to be improved. In this study, a new EEG feature combining the frontal asymmetry and differential entropy features is used to classify negative and positive emotion. The result of our research shows that the combined feature is better than the entropy features in emotion recognition. The average classification accuracies of the frontal asymmetry combined with differential entropy features and entropy feature in our study are $72.1 \%$ and $67.7 \%$ respectively. This result indicated that this combination feature is more suited for emotional classification.

\section{Introduction}

Emotion is vital to humans. It not only helps people communicate, but also plays a key role in rational and intelligent behavior. There are many ways for emotion recognition. Facial expressions as a recognition method are often used [1]. In recent years, other methods have been continuously improved for emotion recognition. Some physiological signals are widely used, such as electrocardiogram (ECG), electromyogram (EMG), electroencephalogram (EEG). Among these, EEG-based emotion recognition plays a very important role, which has received the attention of many researchers $[2,3]$.

The EEG is a direct measurement of neural activity. Researchers have done many works to identify emotions based on the EEG signals. Among them, how to classify human's emotional states is a prime issue.

There are two models describing emotional states, the discrete and the dimensional model. The discrete model includes six basic emotional states, such as anger, disgust, fear, sadness, happiness, and surprise [4]. The dimensional model comes up with the valence-arousal space [5]. The valence and arousal scales represent the valence arousal space for all emotional states. In recent years, based on DEAP database [6], many studies have used the database to classify valence and arousal $[7,8]$.

Davidson used brain asymmetry to analyze EEG [9], which achieved good results and was used widely by other researchers $[10,11,12]$. The entropy feature like approximate entropy (ApEn) and sample entropy (SampEn), as an important feature of emotion recognition, are capable to extract emotional information from complex EEG signals. Seyyed Abed Hosseini uses the approximate and wavelet entropy to analysis emotion state [13] .Yong Zhang et al use the method of empirical mode 
decomposition (EMD) and sample entropy for emotional recognition[14].

In our research, we combine the advantages of asymmetry with entropy information, and put the positive and negative emotions as feature vectors into the support vector machines for classification The results show that the recognition rate of a single subject reaches $92.1 \%$ using the combined features, and the average is about $5 \%$ higher than the use of the different entropy features. In future studies, the combination of frontal asymmetric and different entropy features will have better prospects in emotional recognition.

\section{Materials And Methods}

\subsection{Data acquisition}

The DEAP database recorded 32 participants' EEG and peripheral physiological signals, in which each participant watched 40 one-minute videos. Then the participant performed a self-assessment(SAM) to quantify emotional response to the video after watching each video. The valence model is considered in this study. Valence measures the pleasantness of the stimulus and varies from unhappy or sad to happy or joyful. We only want to study the effects of EEG on emotions, so we extract features from EEG channels.

\subsection{Data selection}

Each video also has a specific value. First, we mark each trail with a positive negative flag according to the valence score. As shown in figure 1(a).The valence score of negative is less than 5 , and if greater than 5,is considered as positive. We selected the data of the SAM valence score of the test and the valence of the original data to make the data more reliable. In addition, because the level of the dominance score determines whether the emotion of the subject is successfully evoked. In general, if the dominance score is greater than 3, the emotion of the subject is aroused [15], so we select out the trial with a dominance score greater than 3 .

The purpose of this study is to prove the role of the frontal for the emotion, so we chose F3 and F4 channels, as is shown in figure 1(b).

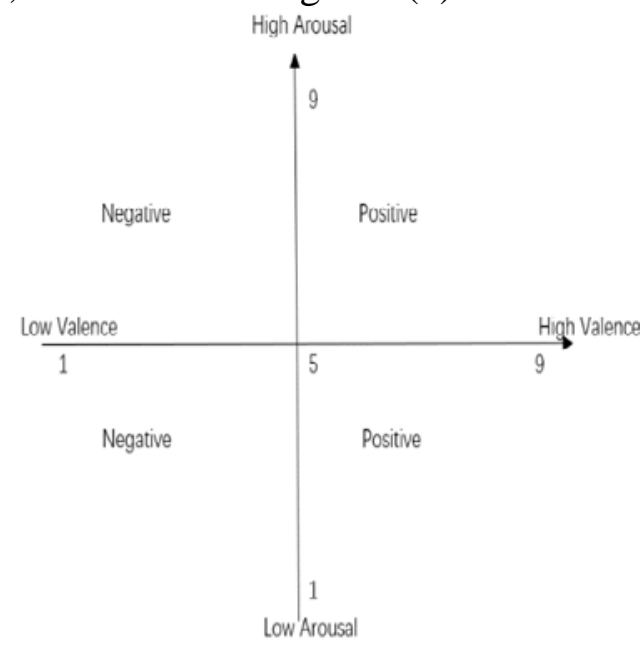

(a)

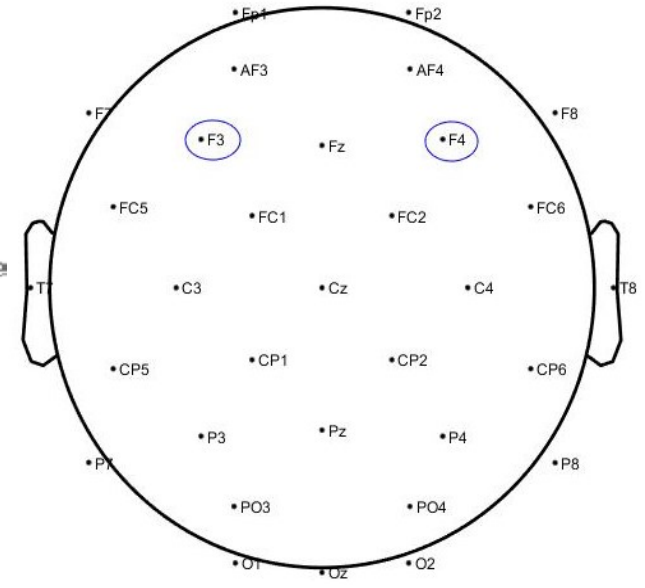

(b)

Figure 1. (a) The relationship between emotion and valence. (b) The map of 32 electrode of the scalp.

Alpha waves are more associated to a aware mental state, and are more visible over the parietal and occipital lobes. Beta waves are related to an active state of mind, more prominent in the frontal 
cortex and over other areas during intense focused mental activity[16].So we choose alpha and beta waves because they are more related to the emotion.

As shown in figure 2, we have compiled a summary of all the data selected by 32 participants, and clearly showed the relationship between the valence and the positive and negative emotions.

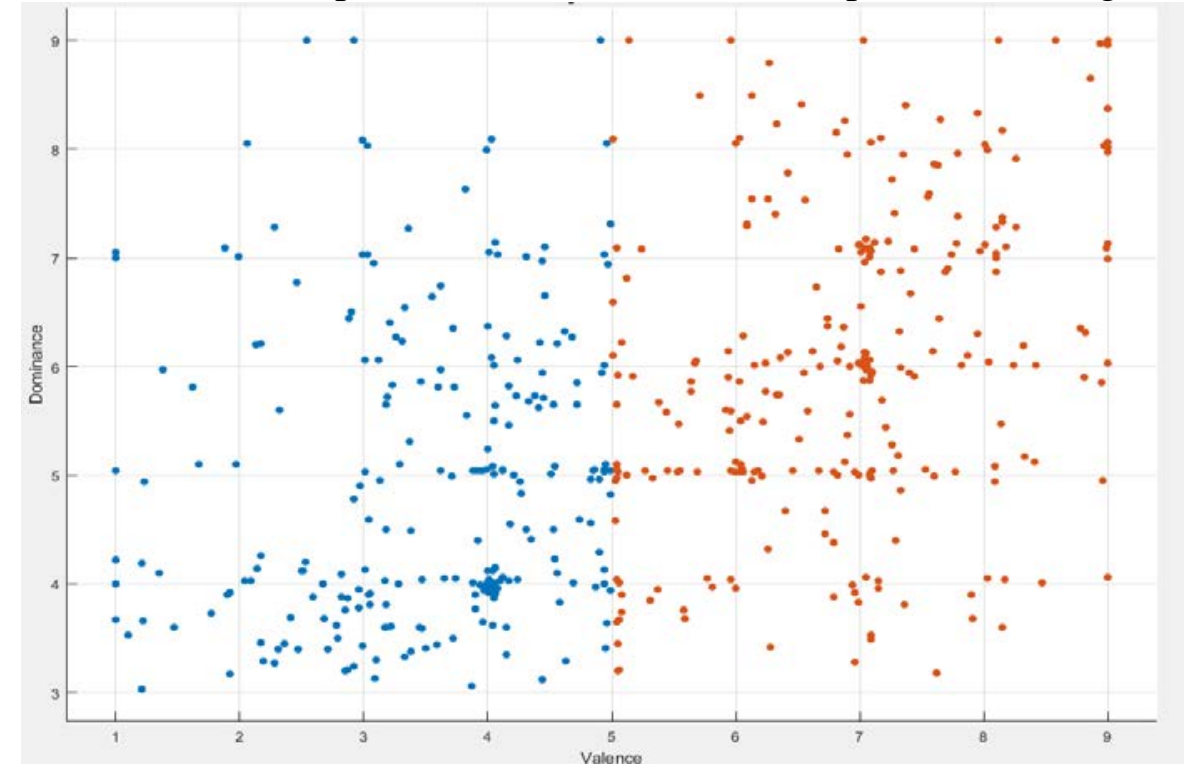

Figure 2. The valence and dominance of all trails, where the blue dots indicate the negative emotions and the orange indicates positive emotions.

\subsection{Feature extraction based on asymmetry and entropy}

\subsubsection{Frontal asymmetry}

To quantify the asymmetry, Davidson uses the asymmetry index DI = (L - R) / $(L+R)$, where L and $\mathrm{R}$ are the powers of the specific bands in the left and right hemispheres, respectively. One type of study suggests that the left frontal is associated with positive emotions, and the right frontal is associated with negative emotions[17,18]. In fact, this theory has been widely accepted and used for research[19,20].

In our study, the F3 and F4 channels of the alpha and beta bands in the frontal were used. Based on the above asymmetry calculation, the logarithm was taken to obtain the asymmetry index, as Fas.

$$
\text { Fas }=\operatorname{In}(F 4)-\operatorname{In}(F 3)
$$

In order to remove the influence of individual differences, we removed the baseline of the resting state for each subject, and then put this into the SVM as a feature vector.

\subsubsection{Approximate Entropy}

Approximate entropy(ApEn) is used to measure the complexity of a signal. The probability of occurrence of a new pattern in a time series is proportional to the complexity. The approximate entropy algorithm is as follows:

(1) Form a vectors $X_{m}(i)=[v(i), v(i+1), \ldots, v(i-m+1)], i=1, \ldots, N-m+1$, and $m$ is a embedding dimension.

(2) Determine the distance as follows

$$
d\left[X_{m}(i), X_{m}(i)\right]=\max (|v(i+k)-v(j+k)|)
$$


(3) Calculate the number of $d[X(i), X(j)]<r$ for all of $i$, and its ratio to $N-m+1$ is defined $C_{i}^{m}(r)$,

$$
C_{i}^{m}(r)=\frac{1}{N-m+1} n u m\left\{d_{m}(X(i), X(j))<r\right\}
$$

Where $r(r>0)$ is a threshold.

(4) Take the logarithm for $C_{i}^{m}(r)$,and then calculate its average for all of $i$,defined as $\phi^{m}(r)$.

$$
\phi^{m}(r)=\frac{1}{N-m+1} \sum_{i=1}^{N-m+1} \operatorname{In} C_{i}^{m}(r)
$$

(5)Increase the dimension to $m+1$,and repeat steps of (1)-(4), we can get a value $\phi^{m+1}(r)$.

(6) Finally define ApEn as:

$$
\operatorname{ApEn}(m, r)=\phi^{m}(r)-\phi^{m+1}(r)
$$

Thus the ApEn is determined by the parameters $m$ and $r$. We choose $m=2$ and $r=0.2 * S D$ in our research, the $S D$ is the standard deviation of the signal.

\subsubsection{Sample Entropy}

The Sample Entropy (SampEn) algorithm as a time-series $\{v(i), 1 \leq i \leq N\}$ of $N$ points of data as follows.

Form a vectors $X_{m}(i)=[v(i), v(i+1), \ldots, v(i-m+1)], i=1, \ldots, N-m+1$, and $m$ is a embedding dimension.

Determine the distance as follows,

$$
d\left[X_{m}(i), X_{m}(i)\right]=\max (|v(i+k)-v(j+k)|)
$$

Where $k=0,1, m-1$.

Define $B_{i}^{m}(r)$ as Given similar tolerancer $(r>0)$, for the number of statistics for each $i$ value $d_{m}(X(i), X(j))<r$, and then calculate its ratio to the total distance $N-m$.

$$
B_{i}^{m}(r)=\frac{1}{N-m} \operatorname{num}\left\{d_{m}(X(i), X(j))<r\right\}
$$

Where $i=1,2, \ldots N-m+1, j \neq i$,num is the number of $d_{m}(X(i), X(j))<r$.

Define the average of the $B_{i}^{m}(r)$ is

$$
B^{m}(r)=\frac{1}{N-m+1} \sum_{i=1}^{N-m+1} B_{i}^{m}(r)
$$

Define $B_{i}^{m+1}(r)$ is the dimension of $B_{i}^{m}(r)$ to $m+1$,and repeat steps (1) to (4).

Define SampEn as

$$
\operatorname{Samp} \operatorname{En}(m, r)=-\operatorname{In} \frac{B^{m+1}(r)}{B^{m}(r)}
$$

We choose $m=2$ and $r=0.2 * S D$ in our research.

\section{SVM}

SVM is a machine learning method which is based on statistical learning theory of VapnikChervonenkis dimension theory [21], mainly solves the small sample problem. For two-class problem, given training $\operatorname{set}\left\{x_{k}, y_{k}\right\}_{k=1}^{N}$, input set $x_{k} \in R^{n}$,output set $y_{k} \in R^{n}$ and $y_{k} \in\{-1,1\}$, we use the following linear classifier,

$$
y_{k}=\operatorname{sign}\left[w^{T} x+b\right]
$$


The nonlinear SVM classifier takes the following form,

$$
y(x)=\operatorname{sign}\left[\sum_{k=1}^{N} \alpha_{k} y_{k} K\left(x, x_{k}\right)+b\right]
$$

Where $\alpha_{k}$ is the Lagrangian multiplier, $K\left(x, x_{k}\right)$ is Kernel function. In our study, we use nonlinear SVM as a classifier, we use the RBF kernel function, which is expressed as follows,

$$
K\left(x, x_{i}\right)=\exp \left(\frac{-\left\|x-x_{i}\right\|^{2}}{2 \sigma^{2}}\right)
$$

Where $\sigma$ controls the width of the kernel function.

\section{Result}

We apply a 128-point window on the pre-processed EEG signal of each channel and each ban to calculate the Fas, ApEn and SanpEn. As shown in Figure 3, the accuracy of the classification of each subject is obtained by comparing the combined feature with the different entropy. For each subject, the accuracy of using the combined feature is higher than the accuracy using the entropy feature. And It can be seen from the figure that the classification accuracy of the 23rd participant is 92.1\% using the combined feature, and the average classification rates of the 32 subjects using the two features are $72.1 \%$ and $67.7 \%$, respectively.

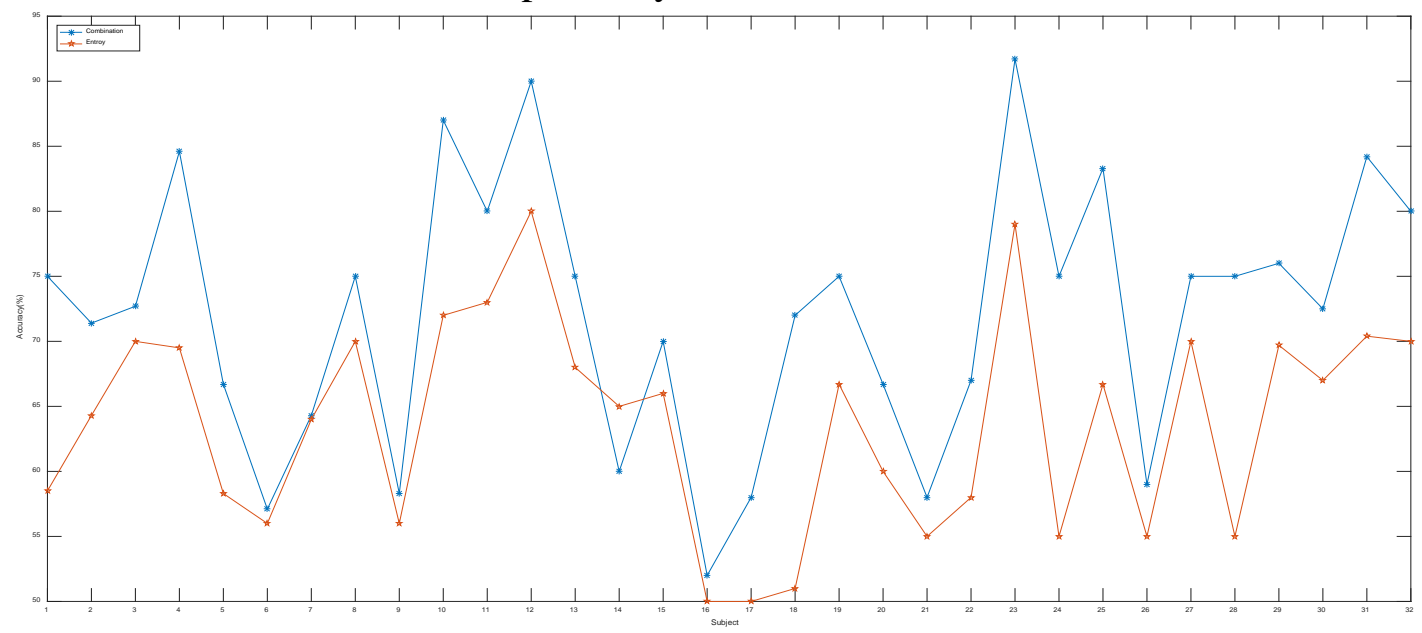

Figure 3 Classification accuracy of 32 subjects use of two features. The blue line indicates the recognition accuracy using the combined feature; the orange line indicates the recognition accuracy using the entropy feature.

\section{Conclusion}

In this paper, a new combined feature is proposed and compared with different entropy. According to the result, the combined feature is more suitable for emotion classification than different entropy. And this new feature increases the stability. At the same time, we can conclude that the frontal asymmetry is a key feature in emotional recognition.

\section{Discussion}

This study used the combined features to identify EEG emotions. And it's also shown that the result of recognition accuracy is improved after adding asymmetric features. The characteristic of entropy is a nonlinear parameter that only to analyze the complexity information of a single channel in a certain band $[22,23]$, but the asymmetry reflects the difference of the emotional changes to the 
left and right hemispheres[24]. So we combine the two features mentioned above, made a result of $72.1 \%$ for valence with 32 participants.

Additionally, for the DEAP database, there are several works that use different features to classify valence. Zheng[25] compared with multiple features, and the result showed that the different entropy as the feature can make for the highest accuracy $69.67 \%$ with 32 participants; Chung's [26] result is $66.6 \%$.In this study, all bands were used to compute the spectral powers of the 93-channel that generated by the 32 traditional channels and 61virtual channels using bipolar montage with 32 participants, Kumar's [27] result is $61.17 \%$ that derived the features of bispectrum for the Fp1 and Fp2 channels of the EEG signals. The comparison with the above study shows that we use the combined feature of frontal asymmetry and entropy feature have a higher recognition rate, which proves that the asymmetry has a important relationship with emotion, because it reflects the common information of the left and right hemispheres.

Therefore, it is also affirmed that the frontal asymmetry is an important feature of emotion recognition. In recent years, prefrontal asymmetry has also been used to analyze mood regulation [28], which will place more emphasis on asymmetry in our future studies of emotional regulation.

\section{Acknowledgements}

This research was supported by the National Natural Science Foundation of China (61472330).

\section{References}

[1] M.J.Black, Y.Yacoob. Recognizing Facial Expressions in Image Sequences Using Local Parameterized Models of Image Motion. International Conf. on Computer Vision, pp. 374-381, 1995.

[2] A. Heraz and C. Frasson, Predicting the three major dimensions of the learner's emotions from brainwaves, Int. J. Comput. Sci., vol. 2, no. 3, pp. 187-193, 2008.

[3] D.O.Bos, EEG-based emotion recognition: The influence of visual and auditory stimuli, Emotion, 2012, $1359: 667$ 670.

[4] P. Ekman, An argument for basic emotions, Cogn. Emotion 6 (3) (1992) 169-200.

[5] A. Mehrabian, Pleasure-arousal-dominance: a general framework for describing and measuring individual differences in temperament, Curr. Psychol. 14 (4) (1996) 261-292.

[6] S. Koelstra, C. Muhl, M. Soleymani, J.S. Lee, A. Yazdani, T. Ebrahimi, T. Pun, A. Nijholt, I.D.E.A.P. Patras, A database for emotion analysis using physiological signals, IEEE Trans. Affective Comput. 3 (1) (2012) $18-31$.

[7] R.Al-Fahad, M.Yeasin, Robust Modeling of Continuous 4-D Affective Space from EEG Recording, IEEE International Conference on Machine Learning \&Applications, 1040-1045, 2017.

[8] M.Ali, AH.Mosa, FA.Machot, K.Kyamakya, EEG-based emotion recognition approach for e-healthcare applications, Eighth International Conference on Ubiquitous \& Future Networks, 2016 :162-164

[9] R. J. Davidson, G. E. Schwartz, C. Saron, J. Bennett, and D. J. Goleman, Frontal versus parietal EEG asymmetry during positive and negative affect, Psychophysiology, vol. 16, pp. 202-203, 1979.

[10] G.L.Ahern, G.E. Schwartz. Differential lateralization for positive and negative emotion in the human brain: EEG spectral analysis. Neuropsychologia 23, 745-755, 1985.

[11] Gotlib, I.H., Ranganath, C., Rosenfeld, J.P., 1998. Frontal EEG alpha asymmetry, depression, and cognitive functioning. Cognition and Emotion 12, 449-478.

[12] W.Heller, The neuropsychology of emotion: developmental patterns and implications for psychopathology, in Psychololgical and Biological Approaches to Emotion, eds N. Stein, B. L. Leventhal, and T. Trabasso (Hillsdale, NJ: Lawrence Erlbaum), 167-211, 1990.

[13] I.J.Seyyed Abed Hosseini, Emotion recognition method using entropy analysis of EEG signals, Image, Graphics and Signal Processing, 2011, 5, 30-36.

[14] Y.Zhang, X.M.Ji, S.H.Zhang, An approach to EEG-based emotion recognition using combined feature extraction method, Neuroscience Letters 633 (2016) 152-157.

[15] X.W.Wang, L.C.Shi, and B.L.Lu, EEG-based Emotion Recognition during Watching Movies Dan Nie, Senior Member ,IEEE Proceedings of the 5th International IEEE EMBS Conference on Neural Engineering Cancun, Mexico, April 27 - May 1, 2011

[16] D.O.Bos, “EEG-based Emotion Recognition: The Influence of Visual and Auditory Stimuli,” Capita Selecta Paper, 
2006.

[17] W.Heller, J.B.Nitschke, The puzzle of regional brain activity in depression and anxiety: the importance of subtypes and comorbidity. Cognition and Emotion 12, 421-447, 1998.

[18] E.K.Silberman, H.Weingartner, Hemispheric lateralization of functions related to emotion. Brain and Cognition 5, 322-353, 1986.

[19] K.Oatley, J.M.Jenkins, Understanding Emotions. Blackwell Publishers Inc., Oxford, England, UK, 1996.

[20] R.B.Zajonc, D.N.McIntosh, Emotions research: some promising questions and some questionable promises. Psychological Science 3, 70-74, 1992.

[21] V.Vapnik, The Nature of Statistical Learning Theory, Springer-Verlag, New York, 1995.

[22] U.R. Acharya, H. Fujita, V.K. Sudarshan, S. Bhat, J.E.W. Koh, Application of entropies for automated diagnosis of epilepsy using EEG signals: a review, Knowledge-Based Syst. 88 (2014) 85-97.

[23] P. Li, C. Karmakar, C. Yan, M. Palaniswami, and C. Liu, Classification of 5-S epileptic EEG recordings using distribution entropy and sample entropy, Front. Physiol. 7 (2016) 136.

[24] PC Petrantonakis ,LJ Hadjileontiadis, A Novel Emotion Elicitation Index Using Frontal Brain Asymmetry for Enhanced EEG-Based Emotion Recognition,IEEE Transactions on Information Technology in Biomedicine A Publication of the IEEE Engineering in Medicine \& Biology Society, 2011 , 15 (5) :737-46.

[25] W.L.Zheng, J.Y.Zhu, and B.L.Lu, Identifying Stable Patterns over Time for Emotion Recognition from EEG, arXiv preprint arXiv: 1601.02197.

[26] S.Y.Chung and H.J.Yoon, "Affective classification using bayesian classifier and supervised learning," in Control, Automation and Systems (ICCAS), 2012 12th International Conference On, 2012, pp. 1768-1771.

[27] N.Kumar, K.Khaund, and S.M.Hazarika, Bispectral Analysis of EEG for Emotion Recognition, Procedia Computer Science 84 ( 2016) $31-35$

[28] D.C.Jackson, C.J.Mueller, I.Dolski, K.M.Dalton, and J.B.Nitschke, Now you feel it, now you don't: Frontal brain electrical asymmetry and individual differences in emotion regulation, Psychological Science, 2010, 14(6):612-617. 